\title{
Las dificultades de lenguaje al comienzo de la escolaridad
}

\section{Geneviève de Weck}

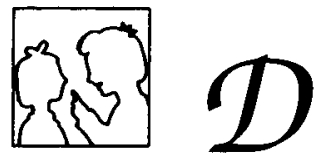

Desde una perspectiva interaccionista del desarrollo del lenguaje, la autora traza la adquisición y el desarrollo del lenguaje oral a través de la adquisición de actividades de lenguaje tales como relatos, narraciones, argumentaciones, etc. adaptadas a las situaciones de comunicación y de interacción en las cuales están inmersos los niños. Intenta ofrecer a los maestros de los primeros años de primaria los instrumentos necesarios que les permitan, por una parte, conocer el desarrollo del lenguaje de sus alumnos reconociendo aquellas verbalizacions "desviadas" pero que son inberentes al desarrollo y, por otra parte, la posibilidad de cambiar sus representaciones, a veces erróneas, sobre lo que se consideran transtornos del desarrollo del lenguaje, es decir transtornos de articulación, retrasos de babla y retrasos de lenguaje. También se ponen de manifiesto las relaciones entre el lenguaje oral y la lectura.

\section{INTRODUCCION}

Cuando los niños empiezan su escolaridad han adquirido unas bases importantes en el ámbito del lenguaje, pero, contrariamente a una idea generalizada, esta adquisición no ha terminado aún, de manera que todavía se observa en los niños pequeños verbalizaciones que no concuerdan con el uso que los adultos hacen de la lengua. Además, si algunos niños continuan su desarrollo del lenguaje sin encontrar demasiados obstáculos, otros, al contrario, sólo lo consiguen con dificultades. Aquellas personas que deben ocuparse de los niños durante este período, en especial los maestros de primaria, deben saber entonces que ciertas verbalizaciones «desviadas» son inherentes al desarrollo, mientras que otras son la manifestación, más o menos grave, de un transtorno del desarrollo del lenguaje. Pero la distinción entre ambas formas no siempre es fácil.

Si pensamos que es importante que los maestros estén atentos a esta dimensión del desarrollo es porque son a menudo los primeros que formulan una perición de consulta ante un especialista (logopeda). En efecto, la entrada en la escuela, al constituir, para un cierto de número de niños, el primer paso hacia una socialización más amplia, puede tener una función reveladora de dificultades a las que, hasta ese momento, la familia no prestó una especial atención. 
Teniendo en cuenta esto, recordaremos brevemente, en la primera parte del artículo, las grandes líneas de la adquisición del lenguaje oral, a la luz de las teorías que nos parecen que actualmente rinden mejor cuenta de dicha adquisición. Evocaremos el desarrollo de la comunicación y el de las capacidades discursivas. Abordaremos también un aspecto más específico del desarrollo, a saber, la evolución del sistema fonológico.

A partir de este repaso, describiremos en la segunda parte, los principales transtornos del lenguaje oral que se observan frecuentemente en esta edad: los transtornos de articulación y de habla que conciernen al sistema fonológico, el retraso del desarrollo del lenguaje que afecta tanto al léxico y a la sintaxis como a las capacidades comunicativas y discursivas. Aunque hagamos hincapié en el lenguaje, no olvidaremos el desarrollo del niño en sui conjunto, puesto que la gravedad de un transtorno del lenguaje es función no tan sólo de los síntomas de lenguaje ${ }^{1}$ observados, sino también de los otros ámbitos del desarrollo (simbolismo, cognición, afectividad, socialización, etc.) y de las condiciones del mismo. Sin embargo, y a pesar de reconocer su importancia, no podremos abordar aspectos que por sí solos requerirían varios artículos. Finalmente, en la última parte, evocaremos brevemente las repercusiones de los transtornos del lenguaje oral en la escolaridad de los niños. Más concretamente, trataremos de mostrar sus relaciones con los comienzos del aprendizaje del lenguaje escrito (sobre todo con la lectura).

\section{LOS PRINCIPALES EJES DEL DESARROLLO DEL LENGUAJE}

A lo largo del presente siglo, el desarrollo del lenguaje ha sido descrito y explicado de diferentes maneras según las corrientes teóricas de referencia de los distintos autores. Los primeros trabajos científicos se deben a lingüistas, psicólogos o médicos que, a partir de la observación de sus propios hijos, escribieron monografías detallando las fases de adquisición de diversas lenguas maternas (por ejemplo, Grégoire, 1937, 1947, para el francés; Leopold, 1939-40) para el alemán y el inglés). Estas monografías son célebres por la finura y la precisión de las observaciones, orientadas tanto a los progresos fonológicos, como al desarrollo de la morfología y del léxico. Dichas monografías presentan, sin embargo, un alcance poco generalizable debido, principalmente, al pequeño número de niños observados y a los problemas metodológicos en el análisis de los corpus recogidos.

En los años 1950, se produjo un importante y decisivo viraje en el estudio del desarrollo del lenguaje gracias a la creación de una nueva disciplina, la psicolingüística, con vocación interdisciplinar. Desde ese momento, los investigadores toman en cuenta al mismo tiempo las aportaciones de la psicología cognitiva (por ejemplo, la corriente piagetiana) y de la lingüística (estructuralismo chomskiano principalmente). No trazaremos de nuevo las diferentes etapas de la psicolingüística del niño (Bronckart, Kail y Noizet, 1983), queremos tan sólo evocar una importante corriente de los años 1970-1980 que ha otorgado importancia a los procedimientos y a las estrategias utilizadas por los sujetos hablantes a partir de indicios que perciben dentro y/o fuera del enunciado. Los investigadores se han interesado particularmente por los mecanismos psicológicos subyacentes en las diversas interpretaciones que sujetos de diferentes edades dan a los mismos enunciados.

Si bien es conocido que los niños no comprenden de entrada los enunciados que les son dirigidos de la misma manera que lo hacen los adultos, esta corriente ha tenido el mérito de precisar las razones de estas diferencias. Por un lado, en el ámbito del léxico, los niños otorgan sentidos diferentes, por sobregeneralizaciones o por infrageneralizaciones (atribución de un sentido más general o más restringido que el 
atribuido por los adultos), tanto a los lexemas nominales como a las unidades léxicas que tienen una función en la organización del discurso, como son los adverbios y las locuciones temporales ${ }^{2}$. Por otro lado, en el campo de la sintaxis, el orden de palabras (Bronckart, Gennari y de Weck, 1981a; 1981b) o el de enunciados es un indicador muy impactante hasta aproximadamente los 5-6 años ${ }^{3}$. Como lo muestran los ejemplos de la nota a final del texto, este indicador se aplica a diferentes estructuras y unidades lingüísticas, lo que le confiere una cierta validez aumentada por la evidencia de su función en varias lenguas.

Actualmente, y desde los años 1980, el interés de los investigadores de numerosas disciplinas de las ciencias humanas se ha dirigido hacia la interacción y la comunicación. En efecto, un gran número de trabajos versan sobre la función de las interacciones en la aparición y el desarrollo del lenguaje y sobre la utilización del lenguaje por los niños en el marco de situaciones de producción finalizadas. Estos trabajos permiten concebir de manera algo distinta la adquisición del lenguaje por los niños, como veremos a continuación.

Bajo el impulso de las propuestas teóricas de Vygotski (1934/1985) (véase también Schneuwly y Bronckart (1985); Vila y Boada (1989); Siguan (1987); Vila, 1987), retomadas más recientemente por Bruner $(1983,1987)$, podemos considerar que la aparición del lenguaje necesita un doble desarrollo durante el primer año de vida de los niños: un desarrollo en el aspecto intelectual y un desarrollo en el aspecto de la comunicación. Si estos dos ámbitos están primero separados (inteligencia preverbal y comunicación pre-intelectual), hacia el año, se fusionan de tal forma que el pensamiento se vuelve verbal y el lenguaje intelectual. Esta posición se opone a la de Piaget (1946/1976, 1946/1970), según el cual, la aparición del lenguaje depende casi exclusivamente del desarrollo cognitivo, es decir que el motor de la adquisición se situa en los mecanismos generales del desarrollo intelectual. Con el advenimiento de la representación, el lenguaje puede aparecer al mismo título que otras conductas simbólicas («hacer como si», juego simbólico, evocar acontecimientos ausentes, etc.). Con estos trabajos, Piaget describió muy bien las etapas de la raíz intelectual del lenguaje.

No obstante, el lenguaje no puede aparecer sin que los niños participen en interacciones. Este aspecto, largo tiempo minimizado, tiene, efectivamente, una función determinante en el desarrollo general de los niños y en particular en el del lenguaje. En efecto, antes de hablar, los niños adquieren progresivamente la experiencia de poder ejercer una influencia sobre los demás (sobre todo comunicación no verbal mediante los gestos y la voz) y de que el lenguaje es un instrumento mediador. Estos descubrimientos se realizan en el marco de interacciones muy ritualizadas y repetitivas entre el niño y el adulto (normalmente la madre), en las cuales cada uno participa activamente en unas actividades rápidamente conocidas por ambos. El adulto crea así unos marcos o formatos (Bruner, 1987) para el niño, que constituyen una especie de mini-cultura representativa de la cultura en la que se integra el niño: aprende no sólo la estructura de la lengua materna (fonología, léxico, sintaxis), sino también los usos del lenguaje y los tipos de discurso producidos en la sociedad.

En efecto, en esta concepción interaccionista del desarrollo del lenguaje, se considera que los niños no deben adquirir unas estructuras lingüísticas como tales, sino unas conductas (Espéret, 1990a) o actividades de lenguaje (Bronckart y col., 1985) tales como la producción de relatos (Fayol, 1985, 1987), narraciones o cuentos (de Weck, 1991), argumentaciones (Rosat, 1991), explicaciones, etc., adaptados a las situaciones en las cuales deben producirlos. Las estructuras conocidas de la lengua se integran entonces en la producción de discursos, cuya gestión no se resuelve en un conjunto de frases, sino que necesita unas capacidades generales de elección y de organización del contenido, de planificación, de lexicalización, etc. 
De esta forma, los niños son de entrada considerados como seres que comunican, y desde su más temprana edad, participan en diálogos (François, 1990). Éstos últimos revisten entonces una doble importancia: por un lado, constituyen la primera forma discursiva adquirida y eficaz para comunicar con los demás y, por otro lado, el lugar mismo de la adquisición del lenguaje. Dicho de otro modo, y según la expresión de Vygotski, el lenguaje se adquiere por y para la comunicación.

Durante los primeros años de vida (hasta los 5-6 años, más o menos), los niños desarrollarán sus capacidades de lenguaje en el marco de diálogos. Es también en este marco que, gracias a las preguntas, a las peticiones de aclaraciones de interlocutores más competentes (niños o adultos), los niños llegarán progresivamente a producir unos discursos menos dialogados, asumiendo una mayor parte de la gestión discursiva. Pensamos en el relato de experiencias personales (Fayol, 1987), que a partir de los 4 años, los niños empiezan a producir, pero no de una forma explícita y completa al principio. A diferencia de los temas de los diálogos cotidianos, las narraciones de estos relatos versan sobre acontecimientos pasados, por lo tanto ausentes, en principio desconocidos del interlocutor. Esta característica supone entonces en el interlocutor, una capacidad para tratar esta asimetría de conocimiento de los temas; hay que esperar hasta los 6-7 años para que los niños hayan adquirido una cierta soltura en la producción oral de este tipo de texto, lo que permitirá a su interlocutor comprenderlo bien. De hecho, los niños diversifican poco a poco sus actividades de lenguaje: producen distintos tipos de discurso en función de las necesidades de su creciente vida social, dándose unos objetivos variados para unos interlocutores cada vez más y más variados (familia, maestros, otros adultos, niños de distintas edades, etc.). Por ejemplo, el placer de la narración se adquirirá gracias a la frecuentación de las historias (leídas o contadas por los adultos), los rudimentos de explicación y/o de argumentación en función de los problemas encontrados en las interacciones, y de los modelos que les son ofrecidos, etc.

En resumen, el desarrollo del lenguaje se caracteriza por una diversificación progresiva de las conductas de lenguaje en función de las necesidades interactivas y de las capacidades socio-cognitivas crecientes (Dolz, Pasquier y Bronckart, 1993). Esta diversificación puede sintetizarse de la siguiente manera:

— del dialogismo al monologismo: la responsabilidad de las actividades de lenguaje se comparte primero con al menos un interlocutor (diálogo), luego progresivamente, asumida sobre todo por el único interlocutor (relatos, narraciones «monologadas») según las necesidades;

- de la evocación de contenidos concretos y presentes a la de contenidos abstractos y/o ausentes, pasados: los diálogos entre niños y adultos están mucho tiempo y frecuentemente centrados en unos temas relacionados con el hic et nunc, mientras que la producción de otros tipos de discurso implica la evocación de contenidos que se «desprenden» de ellos;

- de la lengua oral a la lengua escrita: desde los 6-7 años, los niños empiezan a adquirir capacidades discursivas también por escrito que se arraigan con las adquiridas en la modalidad oral, pero necesitan un distanciamiento respecto a la situación de producción (ausencia física del interlocutor que no puede por lo tanto dar un feed-back directo), y por consiguiente un cierto nivel de abstracción y de reflexión metalingüística (Schneuwly, 1988; Rosat, 1990; de Weck, 1991).

Terminaremos esta parte con algunas observaciones concretas a propósito del desarrollo del sistema fonológico que aún está en plena expansión entre los 4 y 6 años, y que choca mucho a aquellos que se ocupan de niños. La adquisición del sistema fonológico de una lengua consiste de hecho en la adquisición de una doble capacidad: por una parte, los niños deben ser capaces de pronunciar todos los fonemas de la lengua objeto (capacidades articulatorias) y, por otra parte, de producirlos dentro 
de unos encadenamientos y unos contextos variables (capacidades fonológicas). Estos dos aspectos suponen que los niños distingan los diferentes fonemas de su lengua (capacidades perceptivas). Los progresos fonatorios no están únicamente relacionados con la maduración muscular y con las crecientes capacidades perceptivas, sino también con las interacciones con el entorno social.

Los trabajos en este ámbito se refieren pues, por una parte, a la evidencia de un posible orden de adquisición de los fonemas, y, por otra parte, a los modos de disposición de los fonemas en secuencias sonoras. Desde el punto de vista de la adquisición de los fonemas, no se ha podido establecer un orden preciso, común a todos los niños que adquieren una misma lengua. Sin embargo, estos trabajos ponen en evidencia algunas tendencias. En continuidad con los sonidos producidos durante el período de las vocalizaciones, los niños mantienen algunos de ellos, aprenden otros y pierden los que no forman parte del sistema fonológico de la lengua objeto. De este modo, hacia los 48 meses, los niños producen generalmente en torno a un $83 \%$ de los sonidos de su lengua. Los fonemas más fácil y rápidamente adquiridos son las vocales orales y las consonantes anteriores - (bi)labiales - los movimientos de las cuales son, además, los más perceptibles, y requieren poca fuerza articulatoria. Más tarde, se observa la producción de fonemas más posteriores, y de aquellos que requieren un reparto de la fuerza articulatoria entre los diversos mecanismos fonatorios (fonemas sonoros en oposición a fonemas sordos). En castellano, las consonantes líquidas y las vibrantes son los fonemas más difíciles (Serra, 1979). En el transcurso de este período de adquisición, cuando uno u otro fonema no puede aún ser producido, o bien se omite (ejemplo: /banco/ por «blanco» ${ }^{4}$, o bien, se reemplaza por otro que tenga ciertas características comunes (ejemplo:/cabayo/ por «caballo»).

Desde el punto de vista del dominio progresivo de los encadenamientos de los fonemas, quienquiera que observa a un niño nota que éste «deforma» las palabras. Análisis precisos han permitido evidenciar errores sistemáticos, observados en la adquisición de diversas lenguas, y llamados «modelos (patterns) de desarrollo» (Locke, 1983). Sin entrar en detalles, damos algunos ejemplos. Una fricativa puede ser reemplazada por una oclusiva (ej: /topa/ por «sopa»). Los grupos consonánticos pueden reducirse, lo cual permite transformar una secuencia VCCV en VCV. Este fenómeno se realiza ya sea por omisión de una de las dos consonantes (ejemplo: /codero/ por «cordero»), ya sea por sustitución de ambas consonantes por una tercera (ejemplo: /fe:d/ en inglés por "speed»), o bien por la añadidura de una vocal intermedia (ejemplo: /cocoderilo/ por «cocodrilo»). De todas formas, los sujetos son capaces de producir los fonemas omitidos o reemplazados cuando éstos aparecen en otros contextos fonológicos.

\section{Transtornos del desarrollo del lenguaje}

La noción misma de transtorno del desarrollo del lenguaje hace referencia a las representaciones que cada uno se construye más o menos intuitivamente sobre las capacidades de lenguaje de los niños de distintas edades, aún sin disponer todavía, como hemos visto anteriormente, de una descripción general y completa de esta adquisición. Las expectativas de los adultos (padres y maestros en particular) se definen en términos de momentos «cruciales», tales como la aparición del lenguaje (hacia los 12-18 meses), la capacidad para producir enunciados simples de varias palabras formalmente bien construidos (hacia los 3 años), y una cierta soltura para conversar y para explicar (desde los 4-5 años). Si estos puntos de referencia tienen, efectivamente, una cierta pertinencia respecto al desarrollo, hemos visto anteriormente que la adquisición de conductas de lenguaje diversificadas es relativamente 
compleja, y se efectua más allá de los 5-6 años. Por otro lado, como en otros aspectos del desarrollo del niño, existen importantes diferencias individuales (Espéret, 1990b), lo que vuelve más compleja la tarea de apreciar y distinguir los usos normales y patológicos del lenguaje. Entre el conjunto de niños que, según una expresión corriente, «hablan mal», existen situaciones muy claras, como por ejemplo la ausencia casi total de lenguaje oral. No será de estos graves transtornos de lo que trataremos, sino más bien de los transtornos menos graves, pero que merecen de todas formas que les dediquemos una atención particular, debido al malestar que pueden provocar en los niños que los padecen, y de las repercusiones que pueden tener sobre su desarrollo general y sobre la calidad de sus interacciones sociales.

Normalmente, se distinguen tres tipos de transtornos del desarrollo del lenguaje: los transtornos de articulación, el retraso del habla y el retraso del lenguaje (Rondal y Seron, 1991). Los dos primeros afectan de manera distinta al sistema fonológico, mientras que el tercero se refiere a toda la esfera del lenguaje (léxico, sintaxis, discurso y pragmática). Durante la primera infancia, pueden aparecer también transtornos del ritmo, como la tartamudez, así como fases de mutismo más o menos importante. Estos transtornos 5 no están necesariamente relacionados con el desarrollo mismo del lenguaje, y pueden aparecer más allá de los 6 años, incluso en la adolescencia o en la edad adulta. Por razones distintas, y según el grado de ininteligibilidad del discurso del niño, el conjunto de estos transtornos puede ocasionar una perturbación de la comunicación con los demás.

Los transtornos de articulación y el retraso del habla consisten en un conjunto de manifestaciones desviadas en la producción de los fonemas de la lengua objeto. La diferencia entre estos dos tipos de transtornos recubre la distinción que hemos mencionado anteriormente a propósito del desarrollo del sistema fonológico. Un transtorno de articulación consiste en la producción alterada de uno o varios fonemas: o bien el sujeto no consigue producirlo(s), o bien lo(s) deforma. En el primer caso, el/los fone$\mathrm{ma}(\mathrm{s})$ incriminado(s) se omite(n) o se sustituye(n) por otro(s).

Ejemplos de omisión: /oja/ por «roja», /abol/ por «árbol», /tato/ por «tractor». Ejemplos de sustitución: /zapo/ por «sapo», /caza/ por «casa», /laz cazaz/ por «las casas»

La mayor parte del tiempo, el sujeto es consciente de la existencia del fonema que no consigue producir, es decir que lo percibe y lo distingue del fonema de sustitución. Retomando el segundo ejemplo de sustitución (cf. supra), el sujeto tendrá los dos signos «casa» y «caza» en su léxico, aunque los produzca de la misma manera. En los casos de deformación, el sistema fonológico está completo, pero comprende ciertas realizaciones que no corresponden a la lengua meta. Dicho de otro modo, el fonema producido no pertenece al sistema. Las deformaciones de los fonemas silbantes (ceceo) representan dificultades articulatorias corrientes. Por otro lado, sea cual sea la manifestación del transtorno, ésta ocurre en todos los contextos fonológicos, como lo muestran los ejemplos anteriormente mencionados. El sujeto ni siquiera llega, en la mayoría de los casos, a producir de forma aislada el fonema en cuestión. Hablamos entonces de costumbre motriz que no ha podido construirse o al menos de forma correcta.

En cuanto al retraso del babla, éste se refiere a dificultades en el desarrollo de la capacidad de producir unos encadenamientos fonológicos según las reglas y las secuencias previstas en la lengua meta. Los niños son capaces normalmente de articular aisladamente los fonemas de su lengua, pero no pueden hacerlo en todos los contextos. Se observan entonces sustituciones, omisiones y/o añadiduras de fonemas. 
Ejemplos: /kren/ por "tren», mientras que la palabra «tela» no sufre ninguna transformación, /poblema/ por "problema» o /esato/ por «exacto», /enecima/ por «encima».

Estas modificaciones ocurren a menudo cuando varias consonantes se siguen en una secuencia (cf. ejemplos). De hecho, uno de los principios que explican estas distorsiones es el de una armonización consonántica, los sujetos intentan producir secuencias sonoras presentando regularidades (VCVC). En el mismo sentido, se observan también influencias de un fonema sobre otro (que lo precede o lo sigue), con el efecto de aumentar o disminuir sus diferencias.

Ejemplo: /bada/ por «baba»,/kokolate/ por «chocolate».

Estos fenómenos, que aparecen la mayor parte del tiempo en uno de los fonemas de una palabra, pueden extenderse a sílabas,

Ejemplo: /sesé/ por «José» donde el número de sílabas de la palabra se mantiene,

O afectar a fonemas pertenecientes a distintas palabras, produciendo secuencias fonológicas sin respetar el límite de las palabras. En estos casos, las palabras pueden ser producidas correctamente cuando están aisladas o integradas en otro contexto.

En resumen, los transtornos de articulación y los retrasos del habla pertenecen al mismo nivel de producción de lenguaje: el sistema fonológico en su conjunto, con posibles repercusiones sobre la inteligibilidad del discurso. El grado de sistematicidad de una modificación es uno de los criterios que permite diferenciar estos dos tipos de transtornos. Además, la terminología utilizada para designarlos refleja una analogía con el desarrollo del lenguaje. En efecto, se utiliza este término porque las manifestaciones de los retrasos del habla se observan también en el desarrollo normal (cf. supra). En este caso, puede constituir un criterio de diferenciación, entre otras cosas, la intensidad de las perturbaciones y/o su persistencia más allá de los 4;6-5 años. Por el contrario, los transtornos de articulación no se observan en el desarrollo normal, salvo, tal vez, en una primera fase de adquisición, cuando ésta aún no se ha estabilizado desde el punto de vista motor; pudiendo aparecer, además, en ese momento.

Veamos ahora los transtornos del desarrollo del lenguaje más generales, los retrasos de lenguaje. Esta expresión refleja la idea de un desfase temporal en la adquisición considerada normal de distintas competencias de lenguaje. Es corriente describir estos transtornos esencialmente desde el punto de vista del léxico y de la sintaxis. Ahora bien, en la perspectiva interaccionista y discursiva en la que nosotras trabajamos (de Weck, 1993), adquirir el lenguaje significa más bien para un niño ser capaz de producir diferentes tipos de discursos adaptados a la diversidad de las situaciones de interacción. Estos discursos comprenden, por supuesto, un gran número de ítems léxicos integrados en estructuras sintácticas, pero éstas son ampliamente dependientes de las estructuras discursivas generales. De ahí que en esta descripción, se abordará, en primer lugar, los aspectos generales de las dificultades de producción, y, en segundo lugar, las características más locales.

Como ya hemos dicho, entre los 4 y 6 años, los niños participan muy activamente en diálogos entre iguales o con adultos. Comienzan también a producir relatos de experiencias personales, narraciones sobre personajes ficticios y a poder transmitir informaciones a su interlocutor que éste no conoce. Ahora bien, a algunos niños les cuesta mucho llevar a cabo estas diversas conductas. No se trata pues de dificultades ocasionales, tales como las que pueden surgirle a cualquier interlocutor ${ }^{6}$, y a fortiori a un niño que no tenga sus competencias completamente estabilizadas. 
Para el diálogo, tomaremos dos ejemplos relacionados con las reglas conversacionales de base (Bange, 1983; McTear, 1985) y más generalmente con la pragmática (McTear y Conti-Ramsden, 1992). Por un lado, puede perturbarse la alternancia de los turnos de palabra, lo cual se manifiesta por un número demasiado grande de solapamientos de las intervenciones de los interlocutores. Este fenómeno ocurre ya sea porque el niño no sabe encontrar el momento oportuno para tomar la palabra, ya sea porque no consigue indicar bien al otro que momentáneamente ha acabado de hablar. Por otro lado, el niño puede no entender todas las funciones de los turnos de palabra (petición, orden, comentario, etc.) de manera que no podrá emitir las respuestas que espera el interlocutor; ya sea una acción o una verbalización. En particular, algunos trabajos han evidenciado dificultades para responder a preguntas (Brinton y Fujiki,1982; Brinton, Fujiki, Frome, Loeb y Winkler, 1986; Hupet, 1990). No se trata de una incomprensión lingüística de la pregunta, sino más bien de un no respeto de la regla conversacional que estipula que una pregunta va seguida, en principio, por una respuesta. En este caso, el interlocutor tiene la impresión que el niño ignora su pregunta. La noción de regla no debe ser interpretada en un sentido normativo, sino como una convención a nivel de las interacciones verbales. Es evidente que un interlocutor puede, por distintas razones, no saber, no poder o no querer responder a una pregunta, pero en ese caso suele hacer saber a su interlocutor que ha comprendido la función de su enunciado.

En el terreno de discursos menos dialogados, tales como el relato y la narración, los niños con retraso de lenguaje producen formas llamadas "primirivas", es decir unos esbozos de relato como los que se observan en los niños más pequeños. Las producciones consisten en un anuncio de noticia, que abarca generalmente un único enunciado referente a un acontecimiento particular. Pero las circunstancias generales y los principales protagonistas no están explicitados, de manera que el relato no es comprensible para aquél que no ha vivido el acontecimiento. El adulto debe entonces hacer todo tipo de preguntas para ayudar al niño a construir un discurso más explícito. Se trata de una dificultad para manejar de forma más autónoma su discurso, para organizarlo en el plano temporal y para establecer relaciones de un enunciado a otro.

A estas características generales se suman a menudo dificultades más locales, que afectan a la producción verbal ${ }^{7}$ incluso de los enunciados sucesivos. Los niños dominan todavía mal la morfosintaxis de su lengua (Gérard, 1991). Ello se manifiesta sobre todo a nivel de los verbos (las terminaciones), de los determinantes y de los pronombres (las distinciones de género y de persona (Schelletter, 1990) y de las marcas de organización del discurso (conjunciones de coordinación y/o de subordinación). Los enunciados que así se producen son yuxtapuestos, a veces incompletos, incluso agramáticos. Es bueno recordar que la adquisición de todos estos elementos sobrepasa el período que aquí nos interesa. Sin embargo, hacia los 5-6 años, los niños dominan, en principio, las bases que les permiten construir discursos accesibles a los demás.

A veces, también aparecen dificultades en el plano léxico (Gérard, op.cit.). Puede tratarse de una no disposición de la palabra o de imprecisiones que contribuyen a volver opaco el discurso de estos niños cuando intentan explicar algo o a transmitir informaciones. Es la manifestación ya sea de un vocabulario relativamente poco extenso, ya sea de una falta de organización, o incluso de una dificultad en el acceso a este léxico. En último caso, los sujetos tienen conocimientos más extensos de lo que pueden exteriorizar. Aunque a veces sea el aspecto cuantitativo lo que se hace más visible en estos transtornos, hay que recordar que a la hora de establecer un diagnóstico en este ámbito mediante pruebas estandarizadas, nos encontramos con el problema de la norma, aunque se hayan tomado ciertas precauciones metodológi- 
cas en el momento de la constitución de la población de referencia. Efectivamente, se sabe que las diferencias socioculturales y regionales se observan especialmente en este aspecto del lenguaje. De ahí que el aspecto cuantitativo no puede constituir el único criterio de evaluación. Más interesante es entonces la organización del léxico en clases semánticas. En efecto, los conocimientos léxicos no se almacenan en la memoria por simple acumulación, sino que se organizan en redes. Los elementos de las clases mantienen relaciones morfológicas y/o de sentido (inclusión, parte/todo, incompatibilidad, identidad, etc.), y también las propias clases están reunidas en redes. La adquisición del vocabulario (Clark, 1993) incluye también esta dimensión, que nos parece fundamental.

Nos gustaría terminar esta descripción de los retrasos de lenguaje con dos observaciones. Por un lado, para garantizar la claridad de esta exposición, ha sido necesario hacer distinciones entre los diversos aspectos del lenguaje. Pero hay que recordar que en el momento de la adquisición, todos estos ámbitos están en interacción, y que unos progresos en un ámbito tienen repercusiones en los demás. Del mismo modo, cuando se trata de dificultades, si un ámbito es más problemático que otro, se observarán también influencias sobre los demás, que deberán tomarse en cuenta. Por otro lado, hemos adelantado varias dificultades encontradas por algunos niños en el dominio progresivo del lenguaje. Ello no debe hacer suponer que siempre que hagan uso de la palabra vayan a fracasar, y menos aún que cada niño presente transtornos en todos los ámbitos mencionados. Cada niño ha adquirido unas competencias que le permiten interactuar con los demás y que hay que realzar y favorecer, aunque sus producciones no correspondan a lo que pueda esperarse.

\section{¿Inciden los transtornos del lenguaje oral en el aprendizaje del lenguaje escrito?}

Al término de este artículo, creemos que esta pregunta puede estar en la boca del lector, razón por la cual la planteamos, aunque no podamos ofrecer una respuesta muy detallada, ya que ello merecería una discusión sobre las relaciones entre lenguaje oral y lenguaje escrito, entre lectura y producción escrita en el transcurso del aprendizaje. Nos contentaremos con enunciar algunos principios generales que pueden servir como base para una reflexión más amplia.

Generalmente, ningún transtorno del lenguaje oral en sí mismo impide que un niño aprenda a leer y a escribir. Esta afirmación puede parecer contradictoria con la idea todavía ampliamente extendida, según la cual un niño que no domina bien la lengua oral encontrará inevitablemente importantes dificultades para acceder a la lengua escrita. Demos como prueba, en el aspecto clínico, el número de niños que han aprendido a leer, a pesar de los transtornos del lenguaje oral, y para quienes este aprendizaje ha repercutido positivamente en su dominio de la lengua oral. ¿Como pueden entenderse estos hechos?

Teniendo en cuenta los trabajos de numerosos especialistas, definimos la lectura como una actividad de búsqueda de sentido, en la que se mezclan la elaboración de hipótesis sobre el contenido y la forma del discurso escrito y su verificación en el material gráfico. En esta perspectiva, la actividad de descifrado y/o oralización sirve de control externo tanto para el aprendiz-lector como para el adulto que intenta evaluar sus competencias. ¿Cuáles son entonces las capacidades requeridas para efectuar este tipo de actividades?

Algunos trabajos muestran la función de las representaciones que los niños tienen de la lectura y de lo escrito en general en el momento de este aprendizaje (Chauveau, Rémond y Rogovas-Chauveau, 1993). Por otro lado, son necesarios conocimientos relativos al uso del lenguaje y a los distintos tipos de discurso, así 
como conocimientos más generales del mundo, que permiten, todos ellos, generar unas expectativas frente al texto escrito (Sprenger-Charolles, 1986); se trata en otros términos de diversas capacidades metalingüísticas (Gombert, 1991). Otros autores insisten en la importancia de la conciencia fonológica como previa al dominio de la correspondencia grafemas-fonemas. Pero se sabe también que esta conciencia aumenta al mismo tiempo que el aprendizaje de la lectura. Finalmente, otros trabajos evidencian incluso una interacción entre lectura y producción escrita (Rieben, Meyer y Perregaux, $1989^{8}$, sugiriendo más bien un aprendizaje paralelo que desfasado de estas dos competencias.

¿Qué sucede entonces con los niños que tienen transtornos de lenguaje? Tomemos en primer lugar el caso de aquellos niños que presentan dificultades en el aspecto fonológico (cf. supra, los transtornos de articulación y los retrasos de habla). Es evidente que estos niños oralizarán un texto con las mismas manifestaciones que cuando hablan. Por ejemplo, si la palabra «crema» la pronuncian normalmente /trema/, también lo harán cuando la lean en voz alta, comprendiéndola en ambos casos. Esta modificación del significando no significa que la palabra esté mal leída, ni que el acceso al sentido esté trabado. Al contrario, si la palabra ha sido oralizada de esta forma, está claro que la toma de sentido es anterior y que el sujeto la ha dicho según sus capacidades fonológicas de producción. En muchos casos, la visualización de las palabras escritas genera progresos en el aspecto fonológico, en la medida en que la permanencia de lo escrito permite estabilizar unas distinciones y/o unos encadenamientos de fonemas que eran hasta entonces imprecisos o erróneos.

En cuanto a los niños que presentan un retraso de lenguaje, es más difícil bosquejar un cuadro general, en la medida en que este tipo de transtorno varía mucho de un niño a otro en razón de los diversos aspectos del lenguaje que pueden estar perturbados. Evoquemos, sin embargo, algunas pistas de reflexión. Por un lado, ¿cuáles son las relaciones entre la producción verbal como tal y los conocimientos lingüísticos y metalingüísticos ${ }^{9}$ necesarios para leer? En otras palabras, ¿las capacidades de producción alteradas van a la par con una capacidad metalingüística reducida? A veces éste es el caso, sin que por ello se trate de una relación de causa-efecto; pero los niños pueden manifestar también una cierta conciencia de las dificultades, y reconocer por ejemplo enunciados incorrectos producidos por otros o por ellos mismos cuando éstos se les repiten.

Si los niños tienen dificultades en la elaboración de reflexiones metalingüísticas y en el uso de sus conocimientos, podemos temer incidencias en la capacidad de elaborar hipótesis sobre el texto que tienen que leer, tanto en el ámbito local, es decir a nivel de la morfosintaxis de los enunciados, como en el ámbito más general de la organización de los discursos. Valga recordar que la mayoría de los textos que se proponen a los niños son de tipo narrativo, ámbito que los niños con un retraso de lenguaje todavía no dominan demasiado.

Se deduce de estas consideraciones que es necesario que estos niños acceden a lo escrito de otra manera que la que pueden hacer mediante su propia lectura. Estamos pensando en dos vías complementarias. Por un lado, la lectura de textos por parte de lectores más competentes permitirá al niño construir conocimientos sobre las estructuras de la lengua y sobre su uso. Esta actividad puede tener también una incidencia positiva sobre sus capacidades para producir discursos, ya que los textos leídos pueden funcionar como modelos. Por otro lado, el dictado que el niño hace al adulto $^{10}$ es muy fructífero debido a: una producción oral llevada a cabo más despacio (cf. el adulto tiene que escribir poco a poco); la relectura del texto por el adulto; la posible discusión acerca de la formulación de los enunciados y del texto en general, así como, acerca de la organización del contenido. Estas características del dictado que el niño hace al adulto conllevan un aumento del control externo, al que está 
acostumbrado el niño en el diálogo oral, pero este ejercicio puede ser objeto de una reflexión más profunda debido sobre todo a la permanencia de la lengua escrita, lo que permite, por ejemplo, volver atrás y comparar diversas versiones de un mismo texto. Progresivamente, este control externo debería interiorizarse para poder ser utilizado por el niño cuando, él sólo, esté confrontado a una actividad de lenguaje en la que necesite recurrir a la lectura y/o a la producción escrita ${ }^{11}$.

\section{CONCLUSION}

En este artículo, hemos intentado presentar los principales transtornos del lenguaje que pueden observarse en los niños de entre 4 y 6 años, es decir al principio de su escolaridad. Hemos procurado situarlos en una concepción interaccionista del desarrollo del lenguaje, pero a pesar de ello, han sido inevitables ciertas reducciones y simplificaciones. Finalmente, hemos tratado la cuestión de las relaciones entre transtornos del lenguaje oral y aprendizaje de la lectura. No hemos abordado realmente la cuestión de la ayuda que debe ofrecerse a estos niños, y aunque hayamos adelantado la idea de que estos transtornos no impiden que un niño aprenda a leer y a escribir, es necesario en muchos casos darles una ayuda especializada. El aprendizaje de la lectura no constituye en sí mismo un criterio para definir el momento de la intervención. Esta decisión se basa en un conjunto de consideraciones (tipo y gravedad del transtorno, peticiones de ayuda por parte de los padres y de los niños, contexto general de la familia, el desarrollo del niño en otros ámbitos, etc.).

A modo de conclusión, nos gustaría insistir en una idea clave, a saber, que la adquisición del lenguaje es un todo. Ya se trate de la modalidad oral o de la modalidad escrita, los niños deben adquirir conductas de lenguaje que les permitan gestionar y llevar a cabo interacciones verbales cada vez más diversificadas. Esta noción de diversificación puede parecer contradictoria con la idea de que la adquisición del lenguaje forma un todo. De hecho, no lo es en absoluto. Por un lado, esta idea de «todo» debe comprenderse en el sentido en que los procesos generales y comunes (representación de la situación de interacción, selección del contenido a transmitir, planificación y organización del discurso, etc.) intervienen en distinto grado en todas las conductas de lenguaje, ya sean orales o escritas. Por otro lado, se hacen transferencias de conocimientos y/o de competencias entre los distintos campos de adquisición que pueden distinguirse en el terreno teórico.

En este sentido, el paso de la lengua oral a la lengua escrita representa una etapa suplementaria respecto a las experiencias más dialógicas que anteceden, requiriendo un grado de abstracción respecto a la situación de producción, un aumento del control interno de la actividad propia y un aumento de las capacidades metalingüísticas. Ello puede ocasionar, evidentemente, dificultades a algunos niños, y en particular a aquellos que ya las tienen en la modalidad oral. Pero las actividades escritas pueden tener también una incidencia positiva en las capacidades orales, en la medida en que se trata siempre de un proceso de interacción verbal en el que ambas modalidades se influyen mútuamente.

\section{Notas}

1 El francés distingue entre "linguistique" y "langagier", traducimos "linguistique" evidentemente por lingüístico/a, mientras que "langagier", lo traducimos por "de lenguaje" (por ejemplo, "symptômes langagiers", síntomas de lenguaje; "activités langagières", actividades de lenguaje, etc.)

2 Ejemplo: "mañana" se comprenderá como un momento futuro, pero no limitándose por fuerza al día que sigue al momento del habla. 
3 En las investigaciones sobre las relaciones temporales (Ferreiro, 1971), se ha puesto de manifiesto que el orden de enunciación de las proposiciones es interpretado como el orden de los acontecimientos, incluso en enunciados del tipo "Antes de subir la escalera, el muchacho ha tomado la caja". El orden de palabras ejerce también una influencia en la atribución de la coreferencia en casos de ambigüedad (ejemplo: el caballo empuja al perro y arrolla a la vaca"), en este tipo de enunciados, el primer sustantivo es considerado como el referente del pronombre (véase de Weck (1991) para una síntesis).

4 Los ejemplos escritos entre /.../ remiten a la pronunciación y aquellos escritos entre "..." a la palabra correctamente ortografiada.

5 Por razones de espacio, no abordaremos ni la tartamudez, ni el mutismo. El lector podrá remitirse a Rondal y Seron (op. cit.) y a Pfauwadel (1986).

6 Como muestra François (1990), las interacciones verbales no son siempre satisfactorias y en particular con niños pequeños.

$7 \mathrm{NdT}$. En este caso, traducimos "mise en mots" por producción verbal.

8 De manera general, el lector encontrará en Rieben y Perfetti (1989), en Lecocq (1992) y en Chauveau, Rémond y Rogovas-Chauveau (op.cit.) numerosos artículos que abordan las temáticas actuales sobre la lectura.

9 Se toma este término en el sentido general de reflexión sobre la lengua, cualquiera que sea el nivel de reflexión y el aspecto que se tome en cuenta (fonológico, léxico, sintáctico, discursivo, pragmático).

$10 \mathrm{NdT}$. Traducimos "dictée à l'adulte" por "dictado que el/la niño/a hace al adulto" con la finalidad de que éste último lo escriba. Existen tres modalidades distintas: el dictado que el niño hace al maestro, el dictado entre varios niños y el dictado entre dos niños.

11 El lector podrá referirse también a Schneuwly (1991), que estudia los diálogos de los niños con miras a la producción de diferentes tipos de textos escritos.

\section{Referencias}

BANGe, P. (1983) "Points de vue sur l'analyse conversationnelle", en DRLAV, 29, pp. 1-28

BRINTON, B. y FUJIKI, M. (1982) "Comparison of request-response sequences in the discourse of normal and language-disordered children", en Journal of Speech and Hearing Disorders, 47, pp. 57-62

Brinton, B. y FujIKI, M., FrOME, E., LOEB, D. y WINkIER, E. (1986) "Development of conversational repair strategies in response to requests for clarification", en Journal of Speech and Hearing Research, 29, pp. $75-81$

Bronckart, J.-P., Bain, D., Schneuwly, B., Davaud, C. y Pasquier, A. (1985) Le fonctionnement des discours, Neuchâtel-París, Delachaux y Niestlé

BronCKarT, J.-P., GenNARI, M. de WECK, G. (1981a) "The comprehension of simple sentences: the representative perspective and the communicative perpective", en International Journal of Psycholinguistics, 8(2), PP. 5-29

BronCKART, J.-P., GenNARI, M. y de WeCK, G. (1981b) "The comprehension of simple sentences: ontogenesis of modes of processing in French", en International Journal of Psycholinguistics, 8(4), pp. 121-147

BronCKART, J.-P., KAIL, M. y NoIzET, G. (1983) Psycholinguistique de l'enfant. Recherches sur l'acquisition du langage, Neuchâtel-París, Delachaux y Niestlé

Bruner, J.S. (1983) Le développement de l'enfant: savoir faire, savoir dire, París, Presses Universtiares de France

BRUNER, J.S. (1987) Comment les enfants apprennent à parler, París, Retz.

Chaveau, G., Rémond, M. y Rogovas-Chaveau, E. (Eds.) (1993) L'enfant apprenti lecteur. París, INRP, L'Harmattan.

ClaRK, E.V. (1993) The lexicon in acquisition, Cambridge, Cambridge University Press.

de WECK, G. (1991) La cohésion dans les textes d'enfants, París-Neuchâtel, Delachaux y Niestlé

de WECK, G. (1993) "Langage déviant et orthophonie", en TRANEL, 20, pp. 69-87

DOIZ, J., PASQUIER, A. y BRONCKART, J.-P. (1993) “L'acquisition des discours: Emergence d'une compétence ou apprentissage de capacités langagières?", en Etudes de linguistique appliquée, 92, pp. 40-55

ESPÉRET, E. (1990a) "Apprendre à produire du langage: construction des représentations et processus cognitifs", en Le Franģais dans le Monde, numéro spérial, pp. 8-15

ESPÉRET, E. (1990b) “L'acquisition différencielle du langage”, en REUCHLIN, M., LAUTREY, J., MARENDaz, C. y OHLman, T. (Eds.) Cognition: l'individuel et l'universel, Paris, Presses Universitaires de France, pp. 217-252

FAYOL, M. (1985) Le récit et sa construction, Neuchâtel-París, Delachaux y Niestlé

FAYOL, M. (1987) "Vers une psycholinguistique textuelle génétique: l'acquisition du récit", en PiÉraut-Le BonNieC, G. (Ed.) Connaître et le dire, Bruselas, Mardaga, pp. 223-238

FER REIRO, E. (1971) Les relations temporelles dans le langage de l'enfant, Ginebra, Droz

FrANÇOIS, F. (1990) La communication inégale, Neuchâtel-París, Delachaux y Niestlé

GÉRARD, C.L. (1991) L'enfant dysphasique, Paris, Editions Universitaires 
GOMBERT, J.-E. (1991) “Le rôle des capacités métalinguistiques dans l'acquisition de la langue écrite”, en Repères, 3, pp. 143-156

GrÉGOIRE, A. (1937 y 1947) L'apprentissage du langage let II, París, Ed. Les Belles Letrres

HuPET, M. (1990) "Pragmatique et pathologie du langage", en NESPOULOUS, J.L. y LeCLerQ, M. (Eds.) Linguistique et neuropsycbolinguistique: tendances actuelles, París, Éditions de la Société de Neuropsychologie de Langue Française, Pp. 83-97

LECOCQ, P. (Ed.) (1992) La lecture: processus, apprentissage, troubles. Lille, Presses Universitaires de Lille

LEOPOLD, W.F. (1939-49) Speech development of bilingual child, Evanston, Northwestern (4 Vols.)

LOCKE, J.L. (1983) Pbonological acquisition and change, Londres, Academic Press

McTEAR, M. (1985). Children's conversation, Oxford, Basil Blackwell

McTear, M. F. y CONTI-RAMSDEN, G. (1992) Pragmatic disability in cbildren. Studies in Disorders of Communication, Londres, Whurr Publishers Ltd

PIAGET, J. (1946/1976) Le langage et la pensée, Neuchâtel-París, Delachaux y Niestlé

PIAGET, J. (1946/1970) La formation du symbole chez l'enfant, Neuchâtel-París, Delachaux y Niestlé

PfaUWADEL, M.C. (1986) Etre bègue, Le Hameau, Retz

Rieben, L., Meyer, A. y Perregaux, C. (1989) "Différences individuelles et représentations lexicales: comment des cinq enfants de six ans recherchent et copient des", en LIEBEN, L. y PERFETTI, C. (Eds.) L'apprenti lecteur, recherches empiriques et implications pédagogiques. Neuchâtel-París, Delachaux y Niestlé, Pp. 145-169

RIEBEN, L. y PERFETTI, C. (Eds.) (1989) L'apprenti lecteur, recherches empiriques et implications pédagogiques, Neuchâtel-París, Delachaux y Niestlé

RONDAL, J.A. y SERON, X. (1991) Transtornos del lenguaje. Vol 1 Lenguaje oral, lenguaje escrito, neurología Vol. 2 Tartamudez, sordera, retraso mental, autismo. Vol. 3 A fasias, retrasos del lenguaje, dislexia, Barcelona, Paidós (título original en francés: (1982) Troubles du langage: diagnostic et réeducation, Bruselas, Mardaga)

RosAT, M.-C. (1990) "Pour ou contre une analyse de formes textuelles argumentatives", en TRANEL, 16, pp. 99-112

Rosat, M.-C. (1991) “A propos de réalisations orale et écrite d'un texte argumentatif”, en Etudes de Linguistique Appliquée, 81, pp. 119-130

SCHELLETER, C. (1990) "Pronouns use in normal and language impaired children", en Clinical Linguistics and Phonetics, 4(1), pp. 63-75

SCHNEUWLY, B. (1988) Le langage écrit chez l'enfant, Neuchâtel-París, Delachaux y Niestlé

SCHNEUWLY, B. (1991) "Différences entre les processus de production de trois genres: du dialogue entre énonciateurs au texte écrit", en Repères, 3, pp. 45-65

SCHNEUWLY, B. y BROCNKART, J.-P. (1985) Vygotsky aujourd'bui. Neuchâtel-París, Delachaux y Niestlé

SERRA, M. (1979) "Normas estadísticas de articulación para la población escolar de 3 a 7 años en el área metropolitana de Barcelona", Comunicación presentada en el IHler Congreso Nacional de Psicología de Pamplona

SIGUAN, M. (Coord.) (1987) Actualidad de Lev S. Vigostki, Barcelona, Anthropos

SPRENGER-CHARROLES, L. (1986) "Rôle du contexte linguistique, des informations visuelles et phonologiques dans la lecture et son apprentissage", en Pratiques, 52, pp. 9-27

VILA, I. (1987) La funció semiòtica de la ment, Vic, Eumo

VILA, I. y BOADA, H. (1989) “Vygotsky et l'ontogenèse du langage", en Enfance, 1-2, pp. 67-73

VYGOTSKI, L.S. (1934/1985) Pensée et langage, París, Editions sociales 


\section{Las dificultades del lenguaje al comienzo de la escolaridad Genievè de Weck} CL\&E, 1994, 23, pp. 55-68

Resumen: Contrariamente a una idea bastante extendida, el desarrollo del lenguaje va mucho más allá de los 6 años. Esta edad coincide con el principio de la escolaridad y rambién con una socialización más amplia que la realizada en el ámbito familiar. Al llegar a la escuela y al iniciarse el proceso de lecto-escritura, algunos niños son enviados a los logopedas porque presentan, según los maestros, problemas de lenguaje. Sin embargo, no todas las conductas "desviadas" son una manifestación de un transtorno del lenguaje, aunque la distinción entre ciertas verbalizaciones "desviadas", pero inherentes al propio desarrollo del lenguaje, y los transtornos del mismo no siempre es clara. En la primera parte del artículo, se exponen brevemente las grandes líneas de la adquisición del lenguaje oral, evocando el desarrollo de la comunicación y el de las capacidades discursivas, haciendo una especial mención a la evolución del sistema fonológico. En la segunda parte, se describen los principales transtornos del lenguaje, a saber, los transtornos de articulación y de habla y los retrasos del lenguaje. En la tercera y última parte, se exponen las repercusiones de los transtornos del lenguaje oral en la escolaridad de los niños enfatizando las relaciones entre el inicio del aprendizaje de la lengua escrita y la lectura.

Título original: Les difficultes de langage au début de la scolarité. Traducción de Gemma Bertran y Mercè Pujol con nuestro agradecimiento a Montserrat Castelló por sus correcciones

Datos sobre la autora: Geneviève de Weck es doctora en Ciencias de la Educación por la Universidad de Ginebra y ha trabajado durante muchos años en la unidad de didáctica de las lenguas de dicha universidad. Desde 1987 es encargada de curso de logopedia en la Universidad de Neuchâtel. Trabajó durante muchos años como logopeda en el Servicio Médico-pedagógico de Ginebra. Actualmente, compagina su trabajo de formación de logopedas en la Universidad de Neuchâtel con la investigación en dicho ámbito y con una consulta privada en logopedia.

Dirección:Curso para la formación de ortofonistas (logopedas), Universidad de Neuchâtel, Espace Louis-Agassiz, 1, CH - 2000 Neuchâtel, Suiza.

C PERMISOS PARA CITAR O REPRODUCIR EN OTRAS FUENTES: Se pueden citar libremente hasta 500 palabras. Para reproducir una porción de texto mayor, figuras o ilustraciones, se deberá pedir permiso por escrito a la revista, especificando el uso al que se destina el texto. En todos los casos, se deberá citar el copyright de $C L \& E$. En el caso de artículos o textos que hayan sido a su vez reproducidos en $C L \& E$ los interesados deberán dirigirse tanto a los detentadores del copyright original como a $C L \& E$, en el caso de que se quiera hacer uso de la traducción. FOTOCOPIAS: Para todo lo relacionado con el uso mediante fotocopia del material de esta revista, deberán dirigirse a: CEDRO, C/ José Marañón, 10,3. ${ }^{\circ}$ Izda. Tel. 5941575. Fax 4453567 